\title{
Voltammetric Analysis of Sulfamethoxazole on Disposable Graphite Electrodes ${ }^{\dagger}$
}

\author{
Andreea-Laura Panait *, Iulia Gabriela David, Dana Elena Popa, Mihaela Buleandră, \\ Vasile David and Mihaela Carmen Cheregi \\ Department of Analytical Chemistry, Faculty of Chemistry, University of Bucharest, 90-92 Panduri Av., \\ District 5, 050633 Bucharest, Romania; gabrielaiulia.david@g.unibuc.ro (I.G.D.); \\ dana_lena1978@yahoo.com (D.E.P.); mihaelabuleandra@yahoo.com (M.B.); \\ vasile.david@g.unibuc.ro (V.D.); m_cheregi@yahoo.com (M.C.C.) \\ * Correspondence: andreeapanait262@yahoo.com \\ + Presented at the 15th International Symposium "Priorities of Chemistry for a Sustainable Development" \\ PRIOCHEM, Bucharest, Romania, 30 October-1 November 2019.
}

Published: 12 October 2019

Keywords: disposable pencil graphite electrode; sulfamethoxazole; voltammetry

Sulfamethoxazole (SMX) is a sulfonamide antibiotic used in the treatment of digestive, bronchopulmonary, and urinary tract infections. In pharmaceutical preparations, it is often associated with the antibacterial drug trimethoprim (TMP). Studies have shown that SMX and TMP are among the emerging micropollutants of the aquatic environment leading to high resistance of the bacteria to these compounds [1]. Thus, simple and sensitive methods are required for their quantification in different matrices. The voltammetric measurements are often a better choice even more if cheap, disposable working electrodes, like pencil graphite electrodes (PGE) [2], are used. This work presents the electrochemical behavior of SMX at the PGE and the new developed differential pulse voltammetric method for its determination.

The SMX working solutions were obtained by successive dilutions with the corresponding supporting electrolyte of the daily prepared $10^{-3} \mathrm{M}$ ethanolic stock solution. Voltammetric recordings were carried out on an Autolab PGSTAT 12 electrochemical system equipped with a three electrodes measurement cell (working electrode: PGE) [3] and a PC running GPES 4.9 software.

Using the differential pulse voltammetry (DPV) to study the SMX electrooxidation on the glassy carbon electrode, the Pt electrode and the graphite leads electrode of different hardness $(2 \mathrm{H}$, $\mathrm{H}, \mathrm{HB}, 2 \mathrm{~B}$ and $\mathrm{B}$ ) it was noticed that the best signal was obtained on type $\mathrm{B}$ mines. Electrochemical activation of PGE did not lead to any improvement of the SMX signal. The recording of five repeated cyclic voltammograms has demonstrated that SMX oxidation signal decreased when the scans number increased so it was necessary to use another pencil lead for each voltammetric measurements. The influence of the nature and $\mathrm{pH}$ of the supporting electrolyte emphasized that the highest DPV signal of SMX was obtained in Britton Robinson Buffer (BRB) $\mathrm{pH}$ 7.96. In the diffusion-controlled oxidation process of SMX on PGE $2 \mathrm{e}^{-}$and $1 \mathrm{H}^{+}$are involved. It was shown that SMX is not accumulated by adsorption on the PGE. Under the optimum working conditions (PGE B, BRB $\mathrm{pH}$ 7.96), the DPV oxidation peak of the SMX varied linearly with the analyte concentration in the range $1.0 \times 10^{-5}-1.9 \times 10^{-4} \mathrm{M} \mathrm{SMX}$. The repeatability of the electrode response expressed as percentage relative standard deviation was $5.5 \%$ for a concentration of $4.8 \times 10^{-5} \mathrm{M} \mathrm{SMX}$.

The developed DPV method has applicability in the determination of SMX from pharmaceutical products (tablets) and the PGE is cheap and easy commercially available. 
Acknowledgments: This work was supported by University of Bucharest, research grand number 20045/2018.

\section{References}

1. Mourid, E.H.; Lakraimi, M.; Benaziz, J.; Elkhattabi, E.H.; Legoruri, A. Wastewater treatment test by removal of the sulfamethoxazole antibiotic by a calcined layered double hydroxide. Appl. Clay Sci. 2019, 168, 87-95.

2. David, I.G.; Popa, D.E.; Buleandra, M. Pencil graphite electrodes: A versatile tool in electroanalysis. J. Anal. Meth. Chem. 2017, 2017, 1905968.

3. David, I.G.; Florea, M.A.; Cracea, O.G.; Popa, D.E.; Buleandră, M.; Iorgulescu, E.E.; David, V.; Badea, I.A.; Ciucu, A.A. Voltammetric determination of $\mathrm{B}_{1}$ and $\mathrm{B}_{6}$ vitamins using a pencil graphite electrode. Chem. Pap. 2015, 69, 901-910.

(C) 2019 by the authors. Licensee MDPI, Basel, Switzerland. This article is an open access article distributed under the terms and conditions of the Creative Commons Attribution (CC BY) license (http://creativecommons.org/licenses/by/4.0/). 